\title{
Astrophysical studies in Tajikistan: from traditional approach over modeling to empirical properties
}

\section{G. I. Kokhirova}

Address: Institute of Astrophysics of the Academy of Sciences of the Republic of Tajikistan, Bukhoro 22, Dushanbe, 734042, Tajikistan

E-mail: kokhirova2004@mail.ru

\begin{abstract}
New approach developed in the Institute of Astrophysics, Academy of Sciences of the Republic of Tajikistan, aimed to determination of the relationships between Solar System minor bodies is considered. The method consists of modeling of objects motion in the past or in the future, calculation of theoretical parameters, identification of the theoretically predicted properties with the observed ones. Usage of the method allowed to recognize extinct cometary nuclei among the near-Earth asteroids and to establish the parent bodies of some known asteroid-meteoroid complexes.
\end{abstract}

Keywords: comet, asteroid, meteoroid, orbit, evolution, radiant, meteor shower.

\section{Introduction}

The recognition of the genetic relationships between minor bodies is the main actual problem in the inner Solar System astronomy. The population of minor bodies of the Solar System consists of comets, asteroids, meteoroids (sizes $100 \mathrm{mcm}-10 \mathrm{~m}$ ). Numerous meteoroid streams producing meteor showers observable at the Earth are also constituent part of this population.

Revealing of the Associations between Solar System minor bodies is the main direction of researches in the Institute of Astrophysics of the Academy of Sciences of the Republic of Tajikistan. There are well developed both theoretical and observational bases in the Institute for the resolving of the problem.

Besides, methods and approaches for research, observations, reduction of observational material, interpretation and analyzing of data and results are needed. Methods could be either known or new as well as theoretical or experimental, or complex. All of them should be approved and adopted, i.e. they must prove that the results obtained on the basis of their use are reliable, objective, authentic and do not contradict the principal already established affirmations. 
New approach was developed in the Institute of Astrophysics aimed to determination of the relationships between minor bodies (see e.g. Babadzhanov et al. 2008a, 2008b, 2008c, 2009, 2013, 2017). The method consists of

- modeling of objects motion in the past or in the future,

- calculation of theoretical parameters,

- identification of the theoretically predicted properties with the observed ones.

The approach is innovative because previously only present-day objects' orbits were compared and if they are similar then a conclusion was made on a common origin of bodies. Under this it was not taken into account that the orbits undergo the gravitational and non-gravitational effects, and, consequently, could change greatly with time. As a result, the objects orbits today can differ greatly; however objects are related and in the past have originated from single common body due to its fragmentation. Thus, there was a need to find out which orbits the objects had in the past. So, the means for modeling the orbits of celestial objects on the basis of the laws of celestial mechanics and computational mathematics were produced. The created software for modeling orbits or calculating the evolution of orbits allow us to find the orbits of the body at any time in the past or in the future, taking into account the gravitational perturbation of major planets and their mutual effects.

Before consideration the method and stages of its implementation, it is necessary to give some descriptions of the objects of research.

\section{Comets (Fig.1):}

- Number of discovered: $>3500$

- $\quad$ Sizes: few km

- $\quad$ Composition: frozen gases (volatiles) with dust particles

- $\quad$ Orbits: typical comet-like

- Sources: Kuiper belt and Oort cloud at the distance 750 millions $\mathrm{km}$ from the Sun

- $\quad$ Approaching the Sun comets look like nebular objects, behind which stretches a long tail, thus comets consist of a nucleus, a coma and a tail.

\section{Asteroids (Fig.2):}

- $\quad$ Number of discovered: $>700000$

- $\quad$ Sizes: few $\mathrm{m}$ up to hundreds of $\mathrm{km}$ 
- Composition: stone, iron

- Orbits: typical asteroidal

- Sources: Main belt of asteroids at the distance 345-495 millions km from the Sun

- $\quad$ Asteroids look like stars; there are no nucleus, coma and tail.

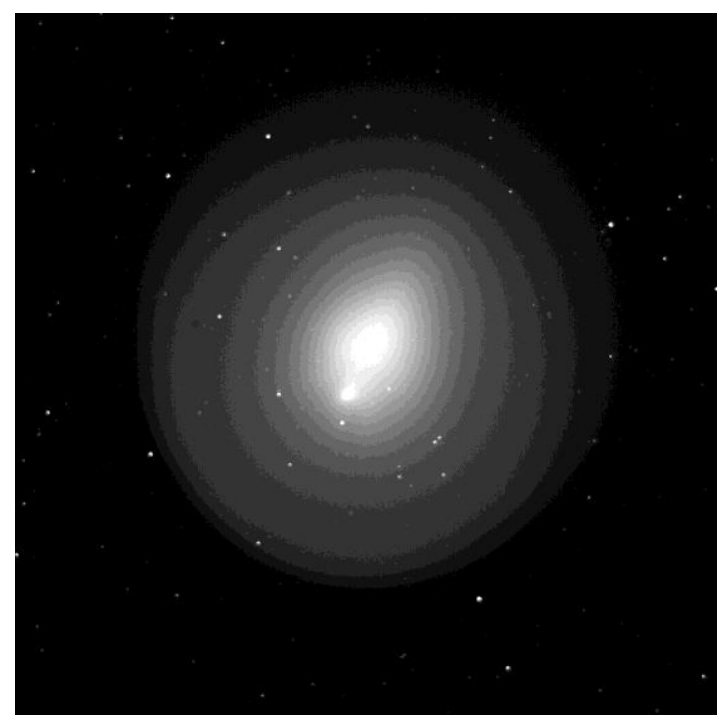

Figure 1.Comet 17P/Holms photographed by the telescope AZT-8 in the Hissar Astronomical Observatory of the IA AS RT.

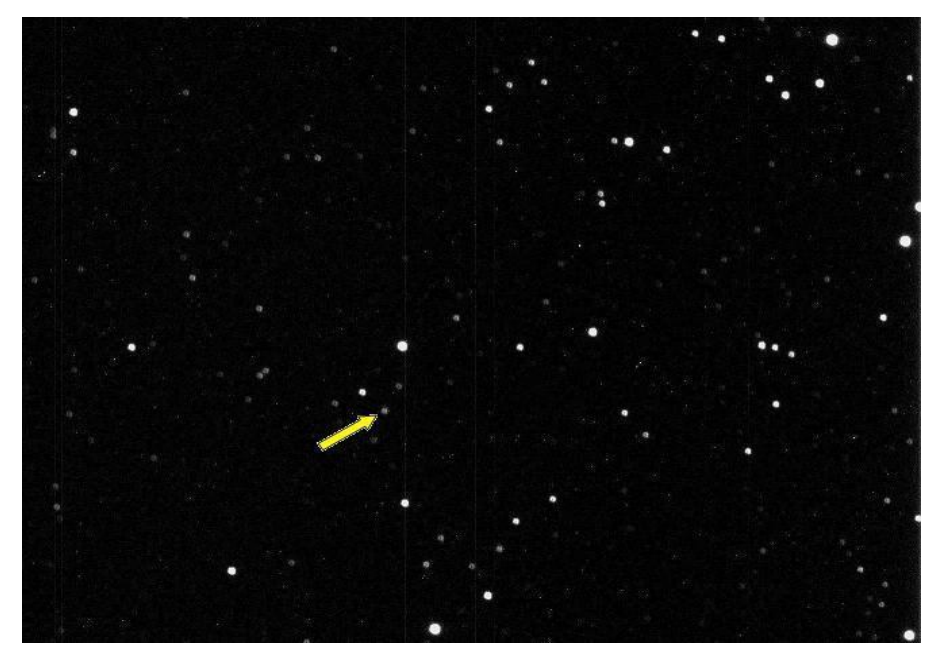

Figure 2. Asteroid 2016LX48 photographed by the telescope Zeiss-1000 in the Sanglokh Astronomical Observatory of the IA AS RT.

Asteroids removed from the Main belt to near-Earth orbits due to perturbations are called Near-Earth Asteroids (NEAs). They located at the distance 195 millions km from the Sun, number of discovered is more than 15 thousands. A 
small fraction in the NEAs population consists of extinct (dead, dormant) cometary nuclei composed of conglomerates of frozen gases and dust particles.

Thus, the properties of the Solar System minor bodies can be summarized as following. Comets and asteroids, as a rule, differ:

- $\quad$ by appearance (for the ground observer);

- by location in the Solar system;

- by orbits;

- $\quad$ by physical properties.

However, by the ground-based observations, extinct comets and asteroids do not distinguish in appearance, they look the same.

\section{Method}

So, criteria are need allowing setting the nature of NEAs and determining the fraction of extinct comets among primordial asteroids. There are few known criteria for distinguishing comets and asteroids. One of them is a classification of object orbit type: comet-like or asteroidal. It is necessary but not sufficient because some comets on asteroidal orbits and asteroids - on comet-like orbits are known at present. Another criterion can be an albedo or reflectivity of the object's surface. The albedo of active cometary nuclei is low (up to 0.12 ), i. e. the nuclei of the comets are very dark. Therefore, we can expect that NEAs having a comet origin have the same albedo. But the definition of the NEAs albedo is a rather difficult problem and today the total number of asteroids with a known albedo moving on comet-like orbits is several dozen.

New approach developed in the Institute of Astrophysics: the existence of an associated meteoroid stream with its observable meteor showers and the comet-like orbit of a NEA are strong indicators of its cometary nature. NEA of cometary origin or an extinct cometary nucleus should form such stream at the period of its past cometary activity. The approach is based on the statement that the formation of meteoroid streams as a result of disintegration of cometary nuclei is undoubted at present. Association of comets and meteor showers has been studies sufficiently well and comets are considering as the main source of meteoroids and streams.

Near-Earth asteroids of cometary origin and meteoroid streams associated with them form complexes of near-Earth objects (NEOs) which have very similar orbits and very likely a common progenitor (Babadzhanov et al. 2015a).

Realization of new approach consists of following stages:

1. Modeling of motion of asteroid or comet, i.e. calculation of the perturbation of the orbital elements during the time embracing one cycle of variations of the 
argument of perihelion $\omega$. Evolution of orbit is calculating using the HalphenGoryachev (Goryachev1937) or Everhart (Everhart 1974) methods. Note for nearEarth objects one cycle of variations of $\omega$ lies in the interval from 3 to 10 thousands yrs, more rare more than 20 thousands yrs.

2. Finding the orbits which are crossing the Earth's orbit. Thus, the number of intersections of the orbits during one cycle of variations of $\omega$ is determined. In most cases, near-Earth objects are quadruple-crossers of the Earth's orbit, namely, for one cycle of changes in the perihelion argument, the object's orbit crosses the Earth's orbit four times. The more rare cases are the octuple intersections.

3. Using found orbits of asteroid (or comet) at the positions of intersections with the Earth' orbit, the theoretical radiants and velocities of meteor showers possibly related with the object under investigation are calculated. For the cases of quadruple crossings we obtain the set of theoretical parameters for four predicted showers.

4. A search for observable showers identical to theoretically predicted showers is carrying out among all published catalogues of meteor showers and individual meteors. If all four theoretical showers simultaneously identified with observable active showers then the related association of these showers and the stream producing them with the object under investigation is considered to be proved.

Application of the method for investigation of the relationship of objects within the $\sigma$-Capricornids complex has given below.

\section{Results}

The asteroid-meteoroid complex $\sigma$-Capricornids consists of five NEAs including (2101) Adonis and 1995CS, and $\sigma$-Capricornids meteoroid stream producing four active meteor showers observable at the Earth (Babadzhanov et al. 2015b). Alternatively 1995CS is classifying as potentially hazardous asteroid. The main properties of Adonis and 1995CS are summarizing in Table 1 (NEOJPL 2016). Here $q$ is perihelion distance, $e$ is eccentricity, $i$ is inclination, $\omega$ is argument of perihelion, $\Omega$ is longitude of ascending node, $\pi$ is longitude of perihelion, and $d$ is diameter.

Comparing the orbital elements of objects the initial supposition appears: a closeness of $q, e, i$, and $\pi$ yielded a suggestion that Adonis and 1995CS were separated at past from a single common body or the smaller 1995CS was fragmented from the larger Adonis. For verification of the suggestion, secular variations of the NEAs orbital elements were calculated by the Everhart method over one cycle of 
variation of the argument of perihelion $\omega$ taking into account the gravitational perturbations from the major planets. As a result of modeling it was found that the time of one cycle of $\omega$ variations is equal to $\sim 13$ kyrs for Adonis and $\sim 11$ kyrs - for 1995CS. During this period both Adonis and 1995CS intersect the Earth's orbit four times under the same values of $\omega$ (Fig.3). The asteroids cross the Earth's orbit four times during these periods, i.e. the heliocentric distances of the ascending $R_{a}$ and the descending $R_{d}$ nodes are equal to $1 \mathrm{AU}$ under the same four values of $\omega: 72^{\circ}, 105^{\circ}$, $253^{\circ}, 287^{\circ}$. Thus, four positions were found when their nodal heliocentric distances are equal to $1 \mathrm{AU}$ that corresponds to positions of intersections with the Earth's orbit. Consequently, related meteoroid streams, associated with each of them, might produce four meteor showers at the Earth.

Table 1. Summary of Orbital (J2000.0) and Physical Properties of the NEAs

\begin{tabular}{|c|c|c|c|c|c|c|c|}
\hline \hline Object & $\begin{array}{c}\mathbf{q} \\
(\mathrm{AU})\end{array}$ & $\mathbf{e}$ & $\begin{array}{c}\mathbf{i} \\
\text { (deg.) }\end{array}$ & $\begin{array}{c}\mathbf{\omega} \\
(\text { deg. })\end{array}$ & $\begin{array}{c}\mathbf{\Omega} \\
(\text { deg. })\end{array}$ & $\begin{array}{c}\boldsymbol{\pi} \\
\text { (deg.) }\end{array}$ & $\begin{array}{c}\mathbf{d} \\
(\mathrm{km})\end{array}$ \\
\hline Adonis & 0.44 & 0.77 & 1.35 & 42.30 & 350.70 & 33.00 & 0.80 \\
1995CS & 0.44 & 0.77 & 2.60 & 252.20 & 135.70 & 27.90 & 0.05 \\
\hline
\end{tabular}

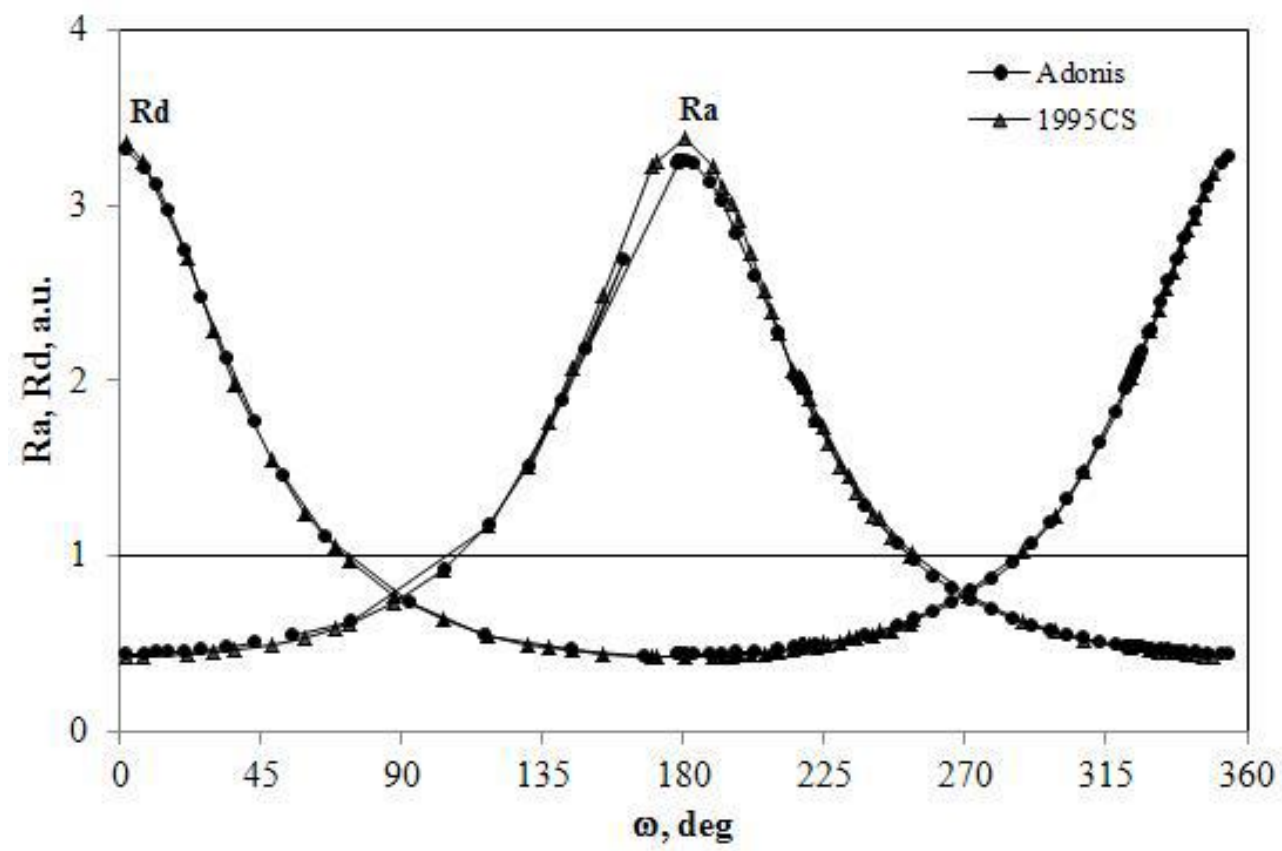

Figure.3. Variations in the heliocentric distances of the NEAs orbits versus the argument of perihelion $\omega$.

Using orbits at these positions the theoretical parameters of four meteor showers associated with each of asteroids were calculated. At the next stage a search for predicted showers in the published data on observable showers and meteors was performed. The theoretical features and observable ones for Adonis are presented in Table 2 and for $1995 \mathrm{CS}$ - in Table 3. In these Tables theoretically predicted 
parameters of four meteor showers denoted as $A, B, C, D$ are given by bold font, Lois solar longitude at the maximum shower's activity, $\alpha_{g}, \delta_{g}$ are the coordinates of geocentric radiant - right ascension and declination, $V_{g}$ is geocentric velocity, $D_{S H}$ is the Southworth and Hawkins criterion (Southworth \& Hawkins 1963), Type indicates Nighttime (N) or Daytime (D) shower, and in the last column the catalogue is given where the observable showers was found - S2,S3 (Sekanina 1973, 1976), MORP (Halliday et al. 1996), PN (McCrosky et al. 1978), TN (Kokhirova \& Babadzhanov 2018). $D_{S H}$ criterion calculated using five orbital elements is the measure of similarity between two orbits. The orbits are considered to be similar if their value of $D_{S H \leq} 0.20$.

Table2. Theoretical and observed orbital elements, geocentric radiants and velocities of meteor showers associated with NEA Adonis (J2000.0)

\begin{tabular}{|c|c|c|c|c|c|c|c|c|c|c|c|c|c|}
\hline $\begin{array}{l}\text { Meteor } \\
\text { shower }\end{array}$ & $\begin{array}{c}\mathbf{q} \\
(\mathrm{AU})\end{array}$ & e & $i^{\circ}$ & $\overline{\boldsymbol{\omega}^{\circ}}$ & $\mathbf{\Omega}^{\circ}$ & $\mathrm{L}_{\Theta^{\circ}}$ & Date & $\alpha_{g}{ }^{\circ}$ & $\delta_{g}{ }^{\circ}$ & $\begin{array}{c}\mathbf{V}_{\mathbf{g}} \\
(\mathrm{km} / \mathrm{s})\end{array}$ & $\bar{D}_{\text {SH }}$ & Type & $\begin{array}{l}\text { Cata- } \\
\text { log }\end{array}$ \\
\hline Theor. A & 0.466 & 0.808 & 7.5 & 281.4 & 106.9 & 106.9 & Jul9 & 293.3 & -13.8 & 26.0 & & $\mathbf{N}$ & \\
\hline -Capds & 0.431 & 0.758 & 2.1 & 290.3 & 107 & 07.6 & 11 & 98.5 & -18.4 & 24.6 & 0.17 & & S2 \\
\hline 57 & 0.543 & 0.810 & 8.4 & 272.1 & 121.8 & 121.8 & Jul24 & 3.0 & $\mid-9.8$ & 24.5 & & & MORP \\
\hline 682 & 0.361 & 0.993 & 12.0 & 285.1 & 103.5 & 103.5 & & 91.4 & -12.8 & 33.6 & & & MORP \\
\hline 793 & 0.557 & 0.726 & 6.7 & 274.2 & 117.6 & 117.6 & & 99.8 & -11.7 & 22.1 & & & MORP \\
\hline 670711 & 0.484 & 0.8 & 7.2 & & 108.7 & 108.7 & & & -14.2 & 27.3 & & & PN \\
\hline 690723 & 0.510 & 0 & 5.5 & & 12 & .7 & & .7 & -12.6 & 23.0 & & & $\mathrm{PN}$ \\
\hline 74 & & & 2.3 & & 10 & $r$ & & & -1 & 21.5 & & & PN \\
\hline 2007 & 0 & 0.8 & 8.5 & & 117 & 117.8 & & 6 & $\mid-11.0$ & 26.6 & & & TN \\
\hline 2307 & 0.499 & 0.7 & 7.8 & 27 & 120 & 120.5 & $\mathrm{Ju}$ & .9 & -10.7 & 25.1 & & & TN \\
\hline 240711 & 0.546 & 0.75 & 7.8 & 27 & 121.1 & 121.4 & Jul24 & 03.6 & $\mid-10.0$ & 23.2 & 0.14 & & TN \\
\hline Theor. B & 0.459 & 0.8 & 5.3 & 10 & 286.3 & 106.3 & Jul8 & 295.1 & $\mid-27.0$ & 26.1 & & & \\
\hline X-Sagds & 0.430 & $0.7 \varepsilon$ & 3.9 & 108.4 & 280.2 & 100.2 & Jul3 & 290.7 & -26.0 & 25.6 & 0.05 & 10 & S3 \\
\hline Theor. C & 0.459 & 0.811 & 5.2 & 77 & 310.5 & 310.5 & Jan31 & 305.4 & -13.7 & 26.0 & & & \\
\hline $\begin{array}{l}\text { Capds- } \\
\text { Sagds }\end{array}$ & 0.414 & 0.758 & 6.2 & 69.8 & 309.8 & 309.8 & $\operatorname{Jan} 31$ & 299.8 & -14.1 & 25.1 & 0.14 & D & S3 \\
\hline D & & 0.0 & 7.5 & & 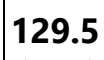 & - & panso & 301.9 & -27.1 & 25.9 & & & \\
\hline & 0.355 & & 6.8 & 242.5 & 145.1 & 325.1 & Feb15 & 314.8 & -23.3 & 26.8 & 0.12 & D & S2 \\
\hline
\end{tabular}

\section{Discussion}

As a result of a search, theoretical showers related both to Adonis and to $1995 \mathrm{CS}$ were identified with the active night-time $\sigma$-Capricornids and $\chi$-Sagittarids, and daytime Capricornids-Sagittarids and $\chi$-Capricornids. These showers are producing by the $\sigma$-Capricornids meteoroid stream. Association with the $\sigma$-Capricornids meteoroid stream and comet-like orbits point to a cometary nature of Adonis and 1995CS which at present are in extinct stage. These objects moving on similar orbits are constituent parts of the $\sigma$-Capricornids asteroid-meteoroid complex, and, very probably, have a common cometary origin, namely, either 1995CS was separated from Adonis, or both 
Adonis and 1995CS were formed as a result of break-up of a large comet-progenitor of the complex which was occurred several tens of thousands years ago.

Table3. Theoretical and observed orbital elements, geocentric radiants and velocities of meteor showers associated with NEA 1995CS (J2000.0)

\begin{tabular}{|c|c|c|c|c|c|c|c|c|c|c|c|c|c|}
\hline $\begin{array}{l}\text { Meteor } \\
\text { shower }\end{array}$ & $\begin{array}{c}\mathbf{q} \\
(\mathrm{AU})\end{array}$ & e & $\mathbf{i}^{\circ}$ & $\boldsymbol{\omega}^{\circ}$ & $\mathbf{\Omega}^{\circ}$ & $\mathbf{L}_{\Theta^{\circ}}$ & Date & $\alpha_{g}^{\circ}$ & $\delta_{g}^{\circ}$ & $\begin{array}{c}\mathbf{V}_{\mathbf{g}} \\
(\mathrm{km} / \mathrm{s})\end{array}$ & $D_{S H}$ & Type & $\begin{array}{l}\text { Cata- } \\
\text { log }\end{array}$ \\
\hline Theor. A & 0.439 & 0.821 & 7.1 & 284.2 & 106.3 & 106.3 & Jul9 & 294.1 & -14.5 & 26.9 & & $\mathbf{N}$ & \\
\hline -Capds & 0.431 & 0.758 & 2.1 & 290.3 & 107.6 & 107.6 & Jul11 & 298.5 & -18.4 & 24.6 & 0.19 & $\mathrm{~N}$ & $\mathrm{~S} 2$ \\
\hline 571 & 0.543 & 0.810 & 8.4 & 272.1 & 121.8 & $\mid 121.8$ & Jul24 & 303.0 & $\mid-9.8$ & 24.5 & 0.12 & $\mathrm{~N}$ & MORP \\
\hline 793 & 0.557 & 0.726 & 6.7 & 274.2 & 117.6 & 117.6 & Jul20 & 299.8 & $\mid-11.7$ & 22.1 & 0.13 & $\mathrm{~N}$ & MORP \\
\hline 670711 & 0.484 & 0.850 & 7.2 & 279.9 & 108.7 & $\mid 108.7$ & Jul12 & 294.7 & -14.2 & 27.3 & 0.05 & $\mathrm{~N}$ & PN \\
\hline 690723 & 0.510 & 0.750 & 5.5 & 278.3 & 120.0 & $\mid 120.7$ & Jul23 & 305.7 & -12.6 & 23.0 & 0.17 & $\mathrm{~N}$ & PN \\
\hline 740707 & 0.465 & 0.670 & 2.3 & 290.7 & $\mid 104.7$ & $\mid$\begin{tabular}{|l|}
104.7 \\
$\mid$
\end{tabular} & Ju & 296.7 & -18.5 & 21.5 & 0.19 & $\mathrm{~N}$ & PN \\
\hline $200710 \mathrm{~A}$ & 0.464 & 0.831 & 8.5 & 281.3 & 117.8 & $\mid 117.8$ & Jul20 & 303.6 & $\mid-11.0$ & 26.6 & 0.1 & $\mathrm{~N}$ & TN \\
\hline 230711 & 0.499 & 0.798 & 7.8 & 278.5 & 120.5 & 120.5 & Jul23 & 304.9 & -10.7 & 25.1 & 0.14 & $\mathrm{~N}$ & TN \\
\hline 240711 & 0.546 & 0.757 & 7.8 & 274.3 & $\mid 121.1$ & $\mid \begin{array}{l}\mid 121.4 \\
\end{array}$ & Jul24 & 303.6 & -10.0 & 23.2 & 0.12 & $\mathrm{~N}$ & TN \\
\hline Theor. B & 0.472 & 0.809 & 4.1 & 100.2 & 290.2 & $\mid 110.2$ & Jul13 & 300.4 & -25.5 & 26.9 & & $\mathbf{N}$ & \\
\hline X-Sagds & 0.430 & 0.783 & 3.9 & 108.4 & 280.2 & 100.2 & Jul3 & 290.7 & -26.0 & 25.6 & 0.05 & $\mathrm{~N}$ & S3 \\
\hline Theor. C & 0.481 & 0.806 & 4.0 & 80.7 & 309.7 & $\mid 309.7$ & $\operatorname{Jan} 30$ & 303.7 & $\mid-15.7$ & 26.9 & & D & \\
\hline $\begin{array}{l}\text { Capds- } \\
\text { Sagds }\end{array}$ & 0.414 & 0.758 & 6.2 & 69.8 & 309.8 & 309.8 & $\operatorname{Jan} 31$ & 299.8 & $\mid-14.1$ & 25.1 & 0.18 & D & S3 \\
\hline Theor. D & 0.488 & 0.804 & 6.9 & 261.6 & 128.9 & 308.9 & Jan29 & 305.1 & -26.6 & 27.1 & & D & \\
\hline X-Capds & 0.355 & 0.789 & 6.8 & 242.5 & 145.1 & 325.1 & Feb15 & 314.8 & -23.3 & 26.8 & 0.11 & $D$ & S2 \\
\hline
\end{tabular}

So, application of new approach to solving the problem of relationship of minor bodies begins from the traditional stage - comparison of present-day orbits, then goes to the stage of modeling the motion of objects in the past and calculations of the theoretical properties of meteor showers, possibly related to the object, and, finally, come to performing of a search and identification of theoretically predicted showers with active and observable ones. Thus the confirmation of the related association between asteroid or comet on the one hand and the streams on the other is finished by the empirical establishment of a nature or an origin of the objects.

\section{Summary}

Using the method, we have already shown that among the known NEAs, the fraction of extinct comets is almost $6 \%$. The parent bodies of some meteoroid streams that were not previously known have been identified among the fraction. The nature of some potentially hazardous asteroids has been established. It is shown that in the streams along with small meteoroids, there are also large bodies with sizes of several 
decameters, entering the Earth's atmosphere; they will produce the phenomena of fireballs or super fireballs.

The method has been approved and adopted; the results have been published in the leading international peer-reviewed journals and are presented at numerous international conferences.

Acknowledgments. We are grateful to the Editorial Board.

\section{References}

Babadzhanov P.B., Williams I.P., Kokhirova G.I. 2008a, MNRAS, 386, 1436

Babadzhanov P.B., Williams I.P., Kokhirova G.I. 2008b, MNRAS, 386, 2271

Babadzhanov P.B., Williams I.P., Kokhirova G.I. 2008c, A\&A, 479, 249

Babadzhanov P.B., Williams I.P., Kokhirova G.I. 2009, A\&A, 507, 1067

Babadzhanov P.B., Williams I.P., Kokhirova G.I. 2013, A\&A, 556, 25B

Babadzhanov P.B., Kokhirova G.I., Obrubov Yu.V. 2015a, Solar System Research, 49,

No.3, 165

Babadzhanov P.B., Kokhirova G. I., Khamroev U.Kh. 2015b, Advances in Space

Research, 55, 1784

Babadzhanov P.B., Kokhirova G.I., Williams I.P., Obrubov Yu.V. 2017, A\&A, 598,94

Goryachev N.N. 1937, Halphen's Method for Calculation of Planetary Secular

Perturbations and its Application to Ceres, Krasnoe Znamya, Tomsk

Everhart E. 1974, Celestial Mechanics, 10, 219

Halliday I., Griffin A.A., Blackwell A.T., 1996, Meteoritics and Planetary Science, 31, 185

NEOJPL 2016, http://neo.jpl.nasa.gov

McCrosky R.E., Shao C.Y., Pozen A. 1978, Meteoritics, 37,p. 44 (in Russian)

Sekanina Z., 1973, Icarus, 18, 253

Sekanina Z., 1976, Icarus, 27, 265

Southworth R.B., Hawkins G.S., 1963, Smith. Contrib. Astrophys., 7, 261 